\title{
EVALUACIÓN DE FUGAS Y PASES EN REHERVIDORES DE PLANTAS DE FRACCIONAMIENTO DE GAS NATURAL
}

\author{
EVALUATION OF LEAKS AND PASSES IN REBOILERS OF NATURAL GAS \\ FRACTIONATION PLANTS
}

\author{
MSc Ing. Carlos Sebastián Calvo ${ }^{1}$
}

\begin{abstract}
RESUMEN
En los últimos años, se han desarrollado diversas técnicas basadas en la aplicación de trazadores y fuentes de radiación para resolver problemas de operación de instalaciones de refinación de petróleo y gas. El objetivo de las experiencias realizadas fue el de identificar fugas y pases, ya sean de fluido térmico o de productos hidrocarbonados líquidos en rehervidores de columnas debutanizadoras, depropanizadoras y despojadoras de columnas de fraccionamiento de nafta-diesel.

Se reporta una serie de determinaciones con radiotrazadores en una planta de fraccionamiento de líquidos del gas natural en Perú para determinar eventuales fugas/pases que podrían existir en los mencionados rehervidores. Para tal fin, se utilizó la técnica de trazadores que ayudaran a detectar en línea, utilizando ácido oleico marcado con I-131 como radiotrazador para la fase hidrocarburo e I-131 en solución para la fase acuosa. Asimismo, se usó un sistema de adquisición de datos, una PC portátil y los detectores en sus respectivas posiciones.

A partir de ese análisis, se registraron valores de fondo de radiación ambiental (línea de base) antes de proceder a cada una de las inyecciones de radiotrazador. Además, se reconocieron contajes de radiación a intervalos de 1 y 2 segundos, dependiendo de cada caso. También, se realizaron 6 inyecciones de trazador, ya sea en tubos o casco de los rehervidores, dependiendo del caso. Así mismo, se percibieron límites de detección de fugas de $0.1 \%$ desde una corriente a otra.
\end{abstract}

Palabras clave: Rehervidor, radiotrazador, detector, fuga/pase, depropanizadora, debutanizadora, columna.

\begin{abstract}
In recent years, it has developed various techniques based on the application of tracers and radiation sources to solve troubleshooting cases for operating facilities in oil and gas refining units.

The objective of the experiments was to identify leaks and passes in reboilers, either thermal fluid or hydrocarbon products in reboilers from a depropanizer, a debutanizer and a naphta-diesel stripping column.

A set of determinations are reported with various radiotracers experiments in reboilers from natural liquid fractioning units in Peru, for determination of leaks/ passes that could exist in three named reboilers.

A tracer technique for online detection was used, by the aid of two radiotracer depending on the phase to be investigated: oleic acid labeled with lodine 131 as a radiotracer for the hydrocarbon phase and an aqueous solution of lodine-131 for the aqueous phase, when necessary. A data acquisition system, a portable PC, and detectors in proper positions were used.

Recording values were synchronized as well as environment background, prior to each injection of radiotracer. Radiation counts were recorded at intervals of 1 or 2 seconds.

Six determinations were conducted by six injection of radiotracer incorporated, either in reboiler tubes or shell, as applicable.

We achieved detection limits leakage of $0.1 \%$, of one stream to another.
\end{abstract}

Keywords: Reboiler, radiotracer, detector, leak/passes, depropanizer, debutanizer, column. 


\section{BASES TEÓRICAS SOBRE LAS DETERMINACIONES REALIZADAS}

\subsection{Funciones de distribución encontradas con auxilio de la tecnología de trazadores}

A continuación, se presentan algunas definiciones cuantitativas de las funciones de distribución de población utilizadas en nuestro estudio.

- El tiempo de residencia de un elemento de la materia que se encuentra en estudio es el que transcurre desde el instante en que el elemento entra en el sistema hasta que lo abandona.

- La edad de un elemento en un determinado instante es el tiempo transcurrido entre la entrada del elemento al sistema y el instante considerado. Este, por supuesto, es menor o igual que el tiempo de residencia.

- La edad es igual al tiempo de residencia para las moléculas que en ese momento están abandonando el recipiente.

- La «distribución del tiempo de residencia» y la «frecuencia de la distribución de edades», para la corriente que sale de un recipiente, son dos nombres diferentes de la misma función $\mathrm{E}(\mathrm{t})$. Esta se normaliza de tal forma que:

$$
\int_{0}^{\infty} E(t) d t=1
$$

El tiempo de residencia se obtiene de la siguiente manera:

$$
E=\int_{0}^{\infty} t \cdot E(t) d t=\frac{V}{Q}
$$

Donde:

$\mathrm{V}=$ Volumen del recipiente

$\mathrm{Q}=$ Descarga

$t=$ Tiempo medio de residencia

V/Q es el llamado «tiempo medio de permanencia» o «tiempo medio de residencia» en el caso de sistemas ideales.

Cuando la señal de entrada de un trazador es un impulso Dirac, como es el caso del esquema de la figura 1, y cuando se trabaja en equipos cerrados, como es el caso de los rehervidores evaluados el presente caso, el tiempo medio se puede obtener a partir de la curva de concentración del trazador a la salida del proceso (curva C).

En la curva adimensional de respuesta $C$ (donde la concentración de referencia c, para el trazador, es la que tendría este si estuviese uniformemente distribuido en todo el recipiente), el área bajo la curva es siempre igual a la unidad. Es decir:

Donde:

$$
\int_{0}^{\infty} \mathrm{C}(\theta) \mathrm{d} \theta=\int_{0}^{\infty} \frac{\mathrm{C}(\theta)}{\mathrm{C}_{0}} \mathrm{~d} \theta=1
$$

$$
\mathrm{C}=\mathrm{c} / \mathrm{c}_{\mathrm{o}} \quad ; \mathrm{y}
$$




$$
\theta=\mathrm{t} / \bar{t} \quad(\text { tiempo adimensional })
$$

Tal que:

$$
\mathrm{C}_{0}=\int_{0}^{\infty} \mathrm{c}(\theta) \cdot \mathrm{d} \theta=\frac{1}{\bar{E}} \int_{0}^{\infty} \mathrm{c}(\mathrm{t}) \cdot \mathrm{dt}
$$

$\mathrm{O}$

$$
\mathrm{E} . \mathrm{C}_{0}=\int_{0}^{\infty} \mathrm{C} . \mathrm{dt}
$$

y teniendo en cuenta la ecuación (1):

de donde:

$$
\begin{aligned}
& \int_{0}^{\infty} E(t) d t=\int_{0}^{\infty} \frac{C(t)}{c_{0} E} d t \\
& E(t)=\frac{1}{C_{0} E} c(t)
\end{aligned}
$$

Sustituyendo (7) en (2):

$$
E=\int_{0}^{\infty} \frac{1}{C_{0} E} c(t) d t
$$

y teniendo en cuenta la ecuación (5):

$$
E=\frac{\int_{0}^{\infty} t . c(t) d t}{\int_{0}^{\infty} c(t) d t}
$$

Se obtiene una ecuación que servirá para determinar el tiempo de residencia en el caso presente.

\subsection{Radiotrazadores en la detección de fugas}

Una fuga es la abertura accidental de una o más partes de un sistema, lo que permite descargas indeseables o entradas de fluidos. Se sospecha de una si hay un comportamiento anormal del sistema, tal como caídas de presión, contaminación del producto o pérdida de eficiencia del proceso. Puede detectarse por varios métodos, con o sin radiotrazador. Algunos se aplican en línea y otros fuera de la línea; a veces, el mismo método se puede usar en ambas situaciones, pero con diferentes sensibilidades. Los que utilizan radiotrazadores son, en su mayoría, útiles para detecciones en línea.

Las técnicas de radiotrazadores para detecciones de fugas se agrupan en dos categorías: 
- $\quad$ Para unidades de procesamiento en planta, recipientes y tuberías, incluyendo aparatos de intercambio de calor, válvulas, calderetas, y redes de distribución de agua caliente industrial.

- Para tuberías enterradas, incluyendo tuberías de transporte de petróleo y gas, redes de distribución de agua, etc.

Con el uso de radiotrazadores, se pueden lograr límites de detección de fugas del $0.1 \%$, de una corriente a otra, mediante la detección externa. Por otro lado, en cuanto al muestreo, se pueden identificar fugas tan pequeńas como del $0.01 \%$ del flujo volumétrico o caudal principal.

En su forma más sencilla, un radiotrazador emisor gamma es inyectado instantáneamente al sistema. El tiempo de tránsito, si hay fuga, se mide colocando detectores de radiación en posiciones definidas y medibles en distancia de uno a otro. El caudal o magnitud de la fuga se calcula conociendo el volumen físico de una sección de tubería entre dos detectores. Este es el caso típico de detección de fugas a través de una línea de bypass.

Por su parte, en las detecciones de fugas a través a través de mediciones de la distribución del tiempo de residencia (DTR), las fugas se pueden encontrar a través del periodo de tiempo que toma el trazador en aparecer por las posiciones de los detectores. Las concentraciones de trazador en las líneas por donde aparece son proporcionales al contaje de radiación. La curva DTR provee indicaciones de fugas si es que estas existen. Ellas se detectan gracias a los picos subsidiarios que preceden al pico principal de la DTR y el caudal de fuga se calcula por la relación de áreas bajo los picos (integración).

En el caso de las evaluaciones que se realizan para evaluar fugas o pases en sistemas de intercambio de calor, las determinaciones se orientan, principalmente, a la determinación y análisis de las curvas de respuesta de un radiotrazador incorporado, ya sea en tubos o casco, según el caso. Esta técnica es muy útil para inspecciones en la industria química y petroquímica y, en general, en aquellas en que se involucran transferencias de calor en sistemas líquido-líquido. El autor ha realizado este tipo de evaluaciones, principalmente, en refinerías de petróleo.

La figura 1 ilustra el principio de la determinación de fugas en intercambiadores de calor. El mismo principio se utiliza para la determinación de eventuales pases de casco a tubos con tan solo modificar el arreglo experimental, tanto de puntos de inyección de radiotrazador como de puntos de detección de este durante su recorrido por el sistema. Así mismo, el principio es aplicable para otras clases de sistemas de intercambio de calor (rehervidores, calderetas, etc.).

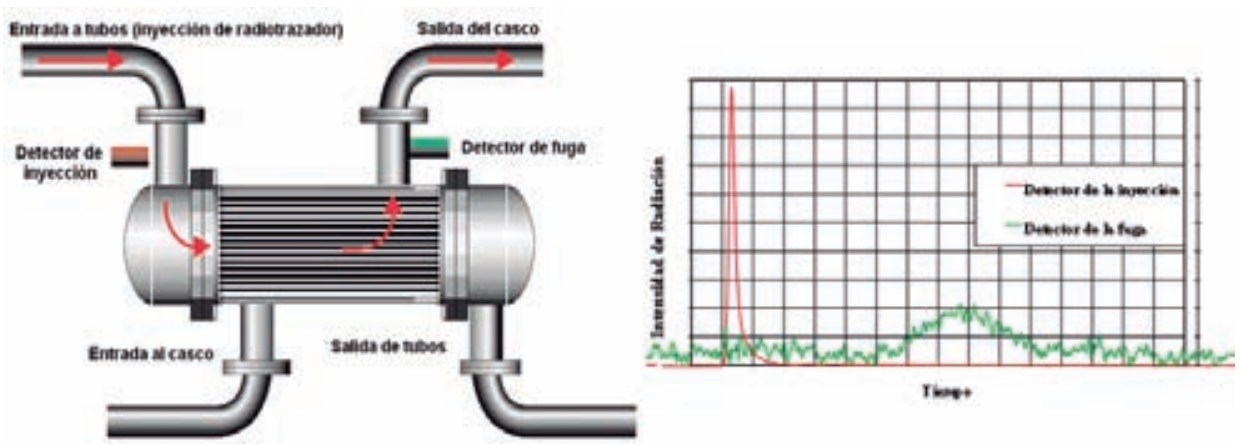

Figura 1. Principio de la determinación de fugas en aparatos de intercambio de calor, utilizando radiotrazadores. Elaboración propia. 


\section{OBJETIVOS DEL ESTUDIO}

Se busca identificar los equipos que presentan fugas o pases, ya sea de fluido térmico o de producto, en el rehervidor de una columna depropanizadora, en el de una columna debutanizadora y en el de una columna despojadora de la fraccionadora nafta-diésel, ubicados en una planta de fraccionamiento de líquidos del gas natural, utilizando técnicas de radiotrazadores.

\section{PROCEDIMIENTO EXPERIMENTAL}

A continuación, se detallan particularidades de la técnica utilizada y los arreglos experimentales para las seis inyecciones de radiotrazador efectuadas para la determinación de fugas o pases en los tres rehervidores evaluados.

Nota: el punto rojo indica el lugar de la inyección del trazador y los puntos azules indican los lugares de posicionamiento de los detectores del trazador (equivalentes a puntos de muestreo).

1.1 PRIMERA EXPERIENCIA: evaluación de fugas de la corriente de fluido térmico que circula por los tubos del rehervidor de la columna debutanizadora hacia la corriente de producto que recorre el casco del rehervidor

El sistema bajo investigación se encuentra delimitado en la circunferencia discontinua de la figura 2.

Inyección del radiotrazador: se realizó en la corriente de fluido térmico therminoil que ingresa al sistema para recorrer los tubos del rehervidor.

Detección en puntos clave del sistema, de acuerdo al detalle siguiente:

Detector 1: al ingreso del fluido térmico al rehervidor

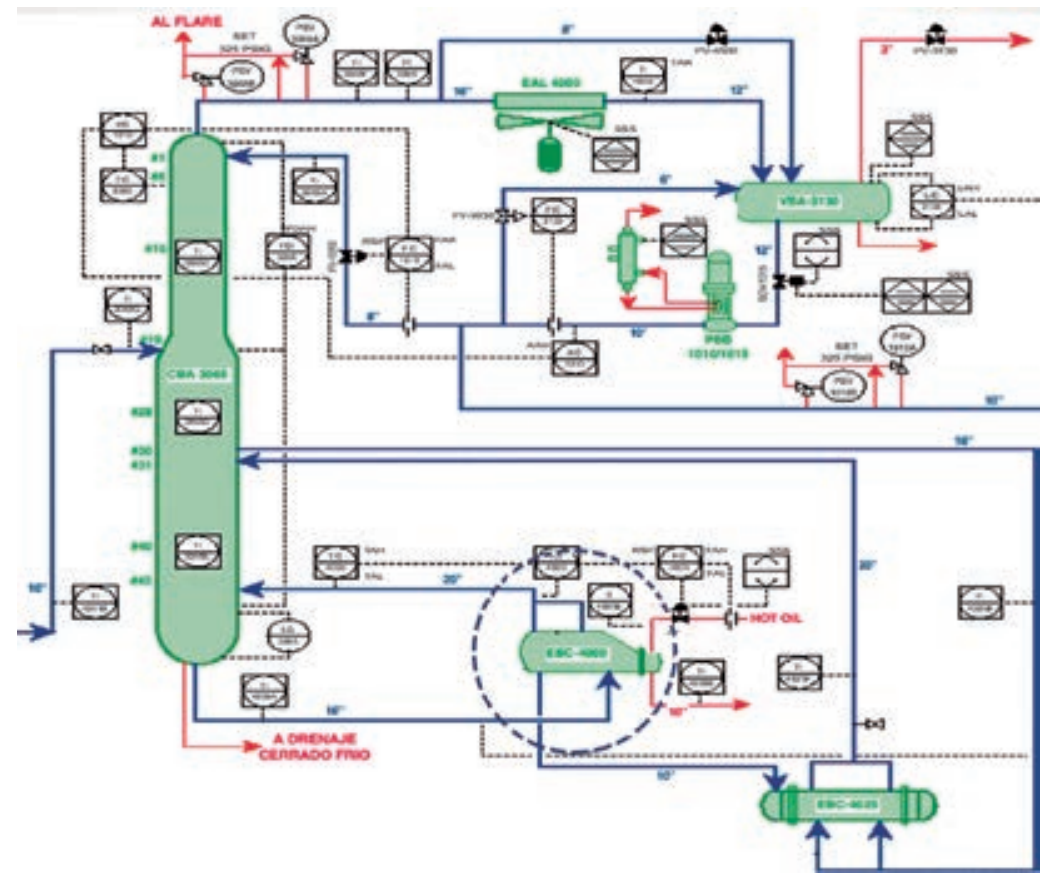

Figura 2. Columna debutanizadora y rehervidor bajo investigación. Elaboración propia. 
Detector 2: a la salida del fluido térmico del rehervidor

Detector 3: en el reflujo de vapor de fondos, del rehervidor a su debutanizadora

Detector 4: a la salida de los fondos del rehervidor

La figura 3 muestra el arreglo experimental.

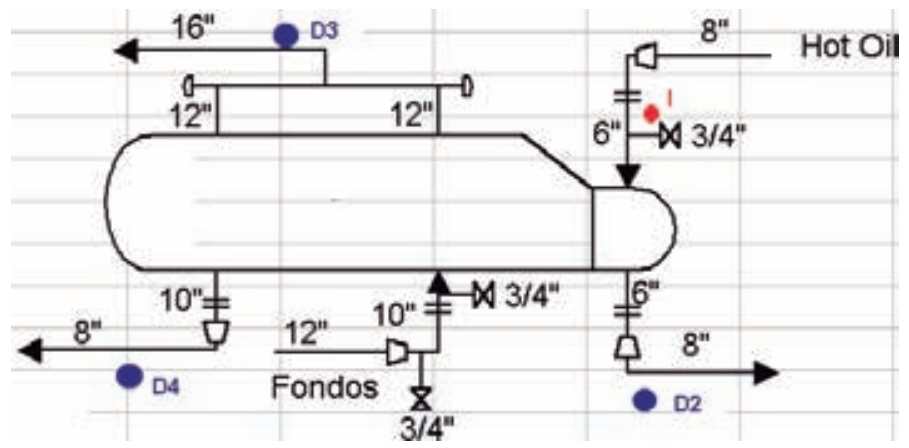

Figura 3. Arreglo experimental para la determinación de posibles fugas de aceite térmico en el rehervidor de la debutanizadora. Elaboración propia.

1.2 SEGUNDA EXPERIENCIA: evaluación de pases de la corriente de producto de la columna debutanizadora que circula por el casco del rehervidor hacia la corriente de fluido térmico que recorre los tubos del rehervidor

Inyección del radiotrazador: se aplica en la corriente de fondos de la debutanizadora que ingresa al sistema.

Detección en puntos clave del sistema, de acuerdo al detalle siguiente:

Detector-1: al ingreso de los fondos de la debutanizadora al rehervidor

Detector-2: a la salida del fluido térmico del rehervidor

Detector-3: en el reflujo de vapor de fondos, del rehervidor a su debutanizadora

Detector-4: a la salida de los fondos del rehervidor

La figura 4 muestra el arreglo experimental.

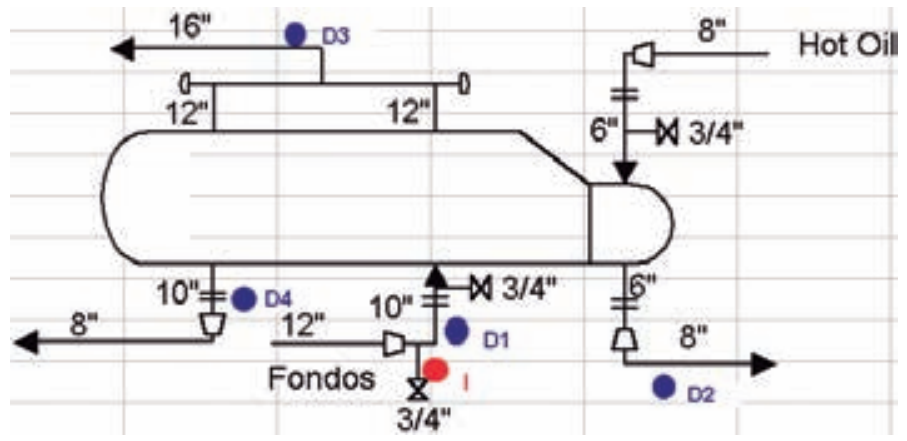

Figura 4. Arreglo experimental para la determinación de posibles pases de producto a los tubos de aceite térmico en el rehervidor de la debutanizadora. Elaboración propia. 
1.3 TERCERA EXPERIENCIA: evaluación de pases de la corriente de producto de la columna depropanizadora que circula por el casco del rehervido hacia la corriente de fluido térmico que recorre los tubos del rehervidor

El sistema bajo investigación se encuentra delimitado en la circunferencia discontinua de la figura 5 .

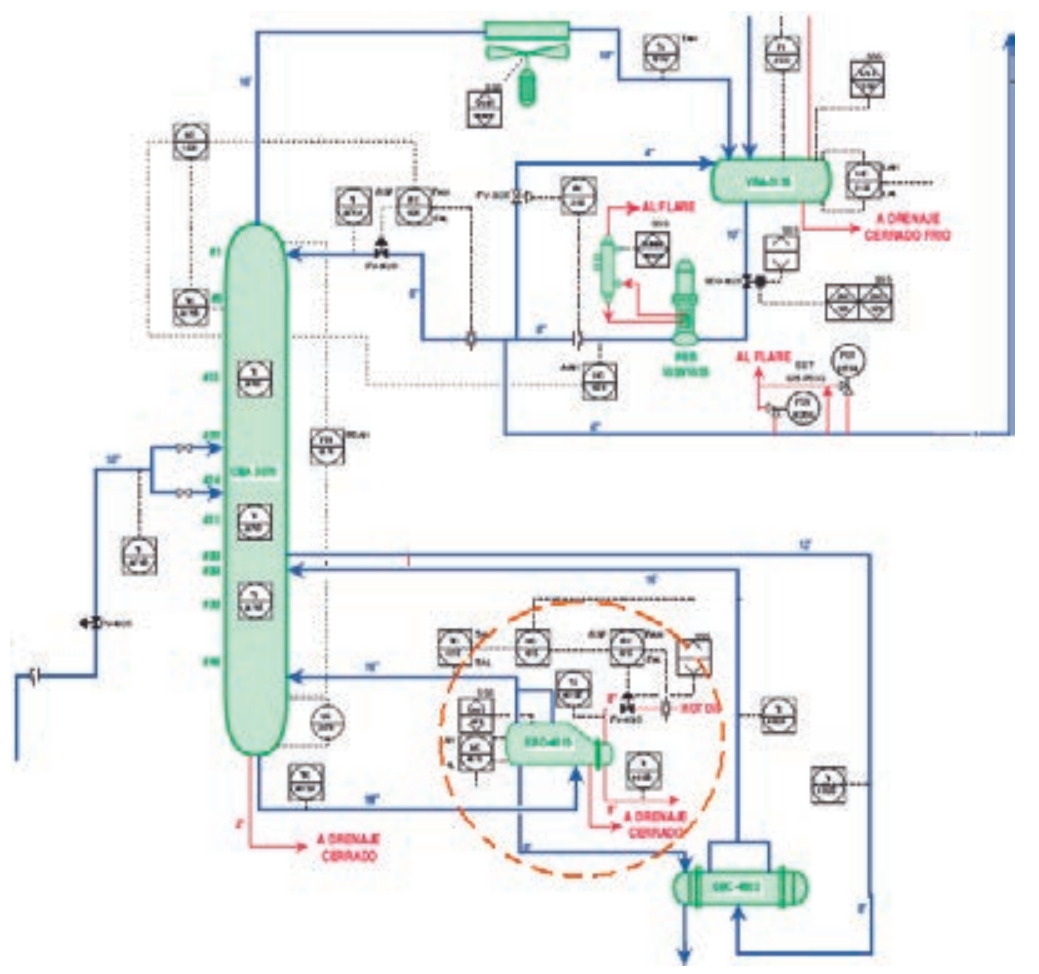

Figura 5. Columna depropanizadora y rehervidor bajo investigación. Elaboración propia.

Inyección del radiotrazador: se aplicó en la corriente de fondos de la depropanizadora que ingresa al sistema.

Detección en puntos clave del sistema, de acuerdo al detalle siguiente:

Detector 1: al ingreso de los fondos de la depropanizadora al rehervidor

Detector 2: a la salida del fluido térmico del rehervidor

Detector 3: en el reflujo de vapor de fondos, del rehervidor a su debutanizadora

Detector 4: a la salida de los fondos del rehervidor

La figura 6 muestra el arreglo experimental. 


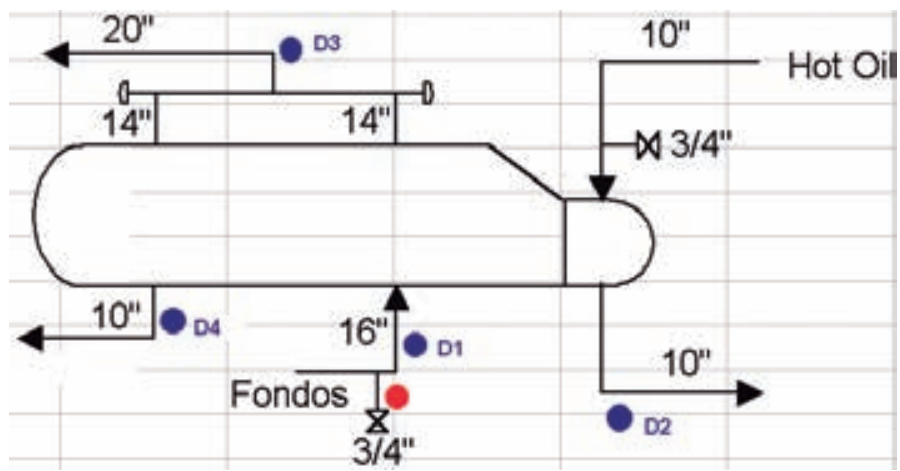

Figura 6. Arreglo experimental para la determinación de posibles pases de producto a los tubos de aceite térmico, en el rehervidor de la depropanizadora. Elaboración propia.

1.4 CUARTA EXPERIENCIA: evaluación de fugas de la corriente de fluido térmico que circula por los tubos del rehervidor de la columna depropanizadora hacia la corriente de producto que recorre el casco del rehervidor.

Inyección del radiotrazador: se aplicó en la corriente de fluido térmico therminoil que ingresa al sistema.

Detección en puntos clave del sistema, de acuerdo al detalle siguiente:

Detector 1: al ingreso de fluido térmico al rehervidor

Detector 2: a la salida del fluido térmico del rehervidor

Detector 3: en el reflujo de vapor de fondos, del rehervidor a su depropanizadora

Detector 4: a la salida de los fondos del rehervidor

La figura 7 muestra el arreglo experimental.

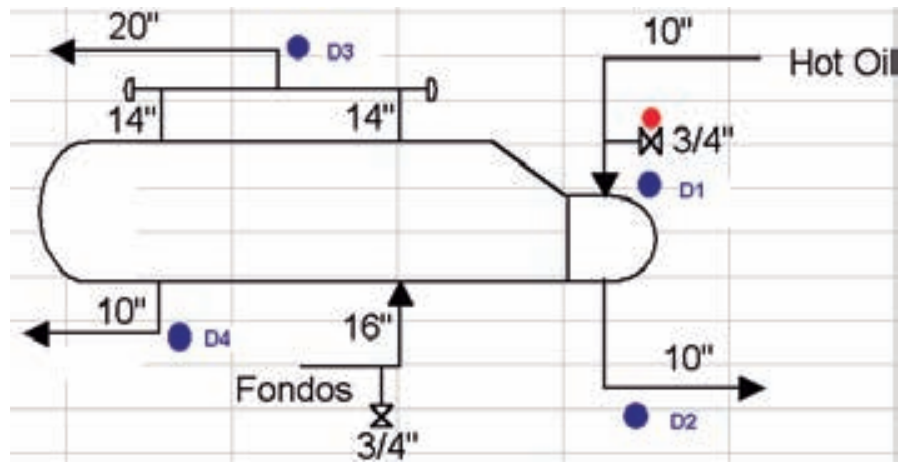

Figura 7. Arreglo experimental para la determinación de posibles fugas de aceite térmico en el rehervidor de la depropanizadora. Elaboración propia. 
1.5 QUINTA EXPERIENCIA: evaluación de pases de fluido térmico que circula por el casco del rehervidor de la torre despojadora de la columna de fraccionamiento nafta-diésel hacia la corriente de diésel que recorre los tubos del rehervidor

El sistema bajo investigación se encuentra delimitado en la circunferencia discontinua de la figura 8 .

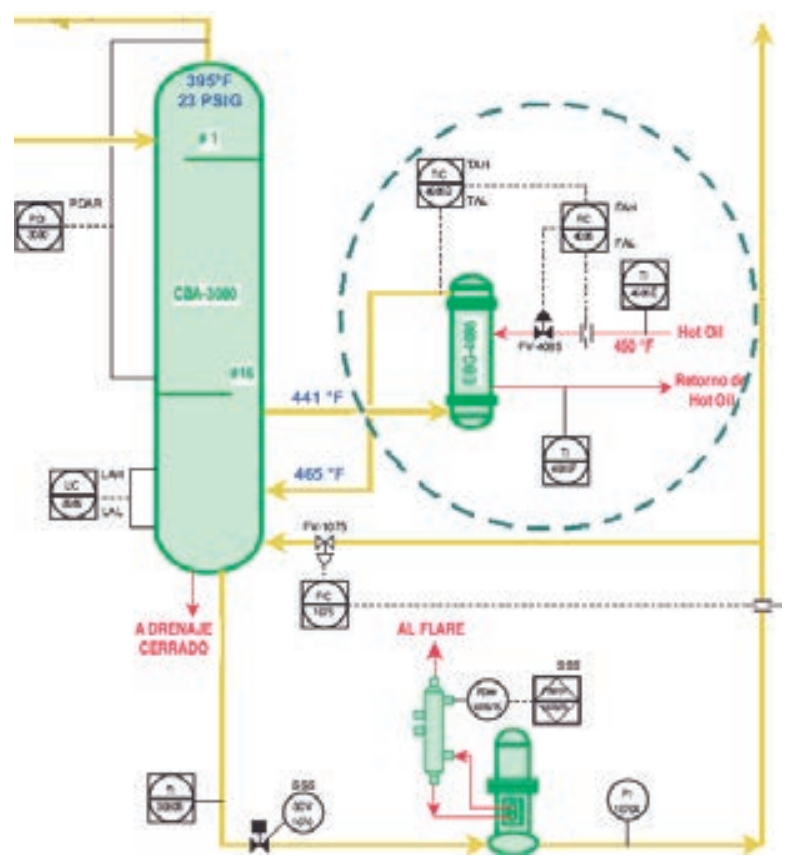

Figura 8. Torre despojadora de la columna de fraccionamiento nafta-diésel y rehervidor bajo investigación. Elaboración propia.

Inyección del radiotrazador: se aplicó en la corriente de fluido térmico therminoil.

Detector 1: al ingreso de fluido térmico al rehervidor

Detector 2: a la salida del fluido térmico del rehervidor

Detector 3: a la salida de la corriente de diésel que retorna del rehervidor a la despojadora de la columna de fraccionamiento nafta-diésel

La figura 9 muestra el arreglo experimental. 


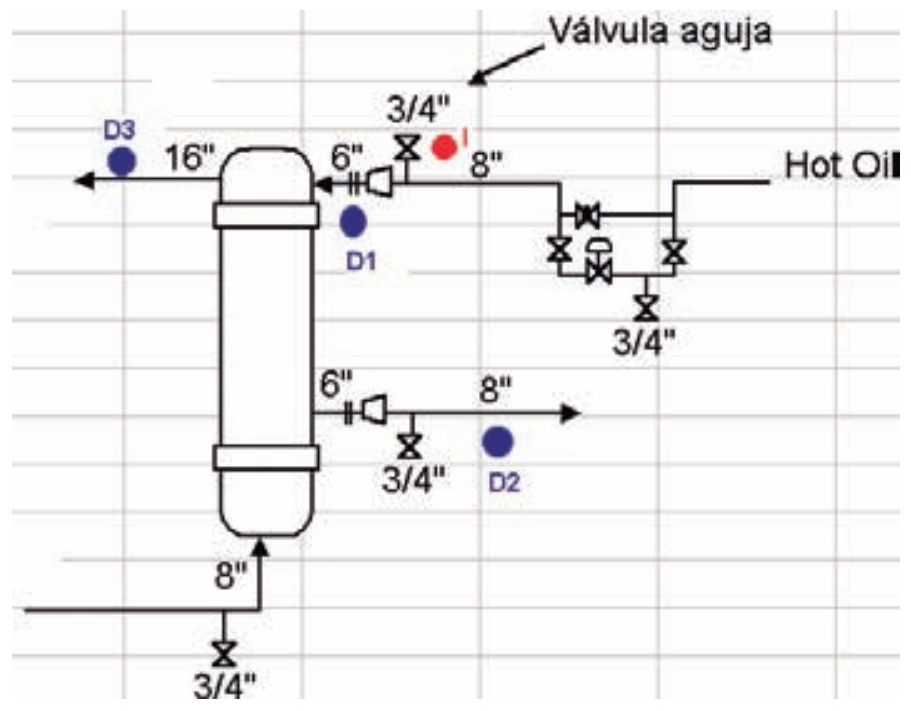

Figura 9. Arreglo experimental para la determinación de posibles pases de aceite térmico en el rehervidor de la despojadora de la columna de fraccionamiento nafta-diésel hacia los tubos de circulación de diésel. Elaboración propia.

3.6. SEXTA EXPERIENCIA: evaluación de fugas de diésel que circula por los tubos del rehervidor de la torre despojadora de la columna de fraccionamiento nafta-diésel hacia la corriente de fluido térmico que recorre el casco del rehervidor

Inyección del radiotrazador: se aplicó en la corriente de diésel que ingresa al rehervidor.

Detector-1: Al ingreso de diésel al rehervidor

Detector-2: A la salida del fluido térmico del rehervidor

Detector-3: A la salida de la corriente de diésel que retorna del rehervidor a la despojadora de la columna de fraccionamiento nafta-diésel

La figura10 muestra el arreglo experimental. 


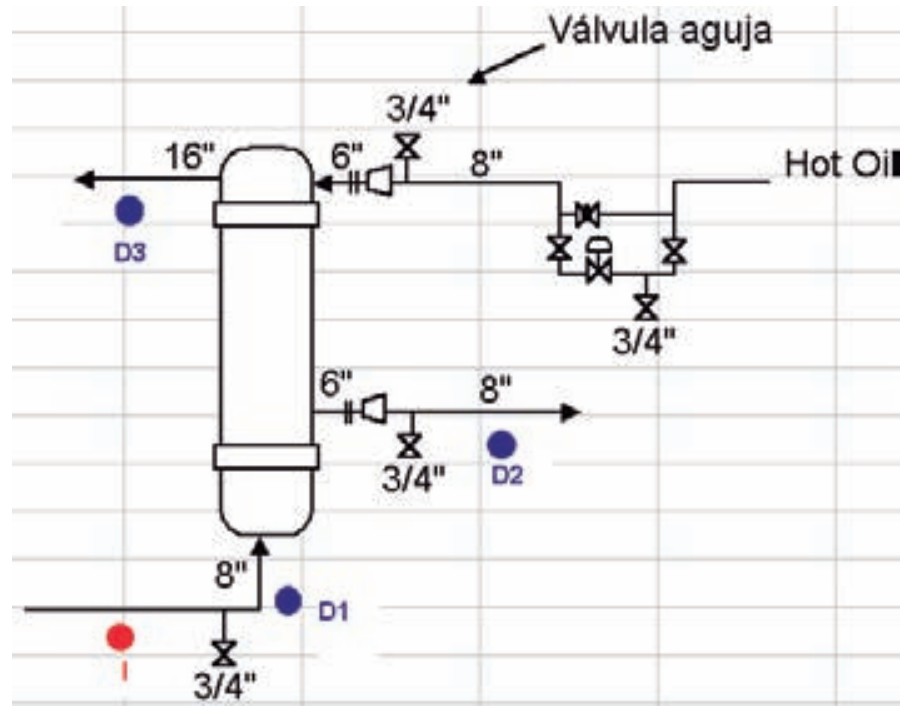

Figura 10. Arreglo experimental para la determinación de posibles fugas de diésel en los tubos del rehervidor de la despojadora de la columna de fraccionamiento nafta-diésel hacia la corriente de aceite térmico que circula por el casco. Elaboración propia.

Nota: el sistema de inyección consistió en una bomba neumática de inyección con sistema de recirculación y un cilindro que contenía diésel. Todo el equipo se montó sobre una plataforma rodante para facilitar su traslado a los diferentes puntos de inyección.

\section{RESULTADOS OBTENIDOS}

A continuación, se presentan las diferentes curvas de respuesta de radiotrazadores obtenidas en los diferentes puntos de inyección y detección para cada uno de los rehervidores evaluados.

\subsection{REHERVIDOR DE LA COLUMNA DEBUTANIZADORA}

\subsubsection{Primera inyección}

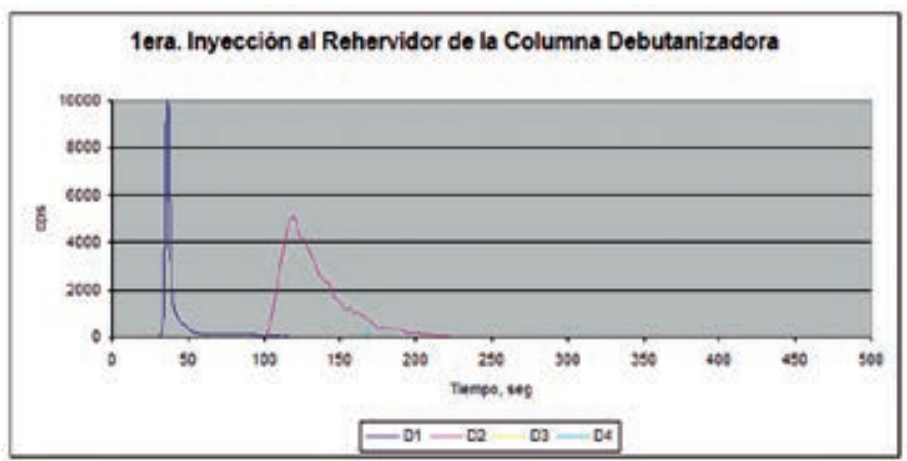




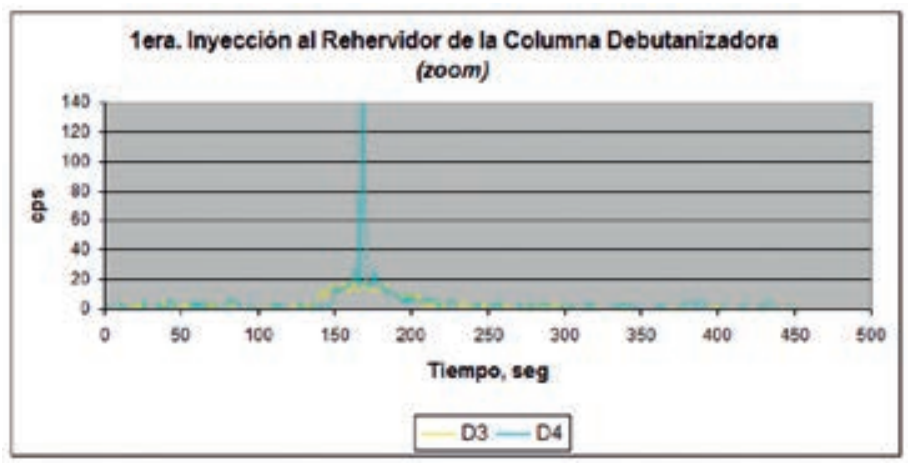

4.1.2. Segunda inyección
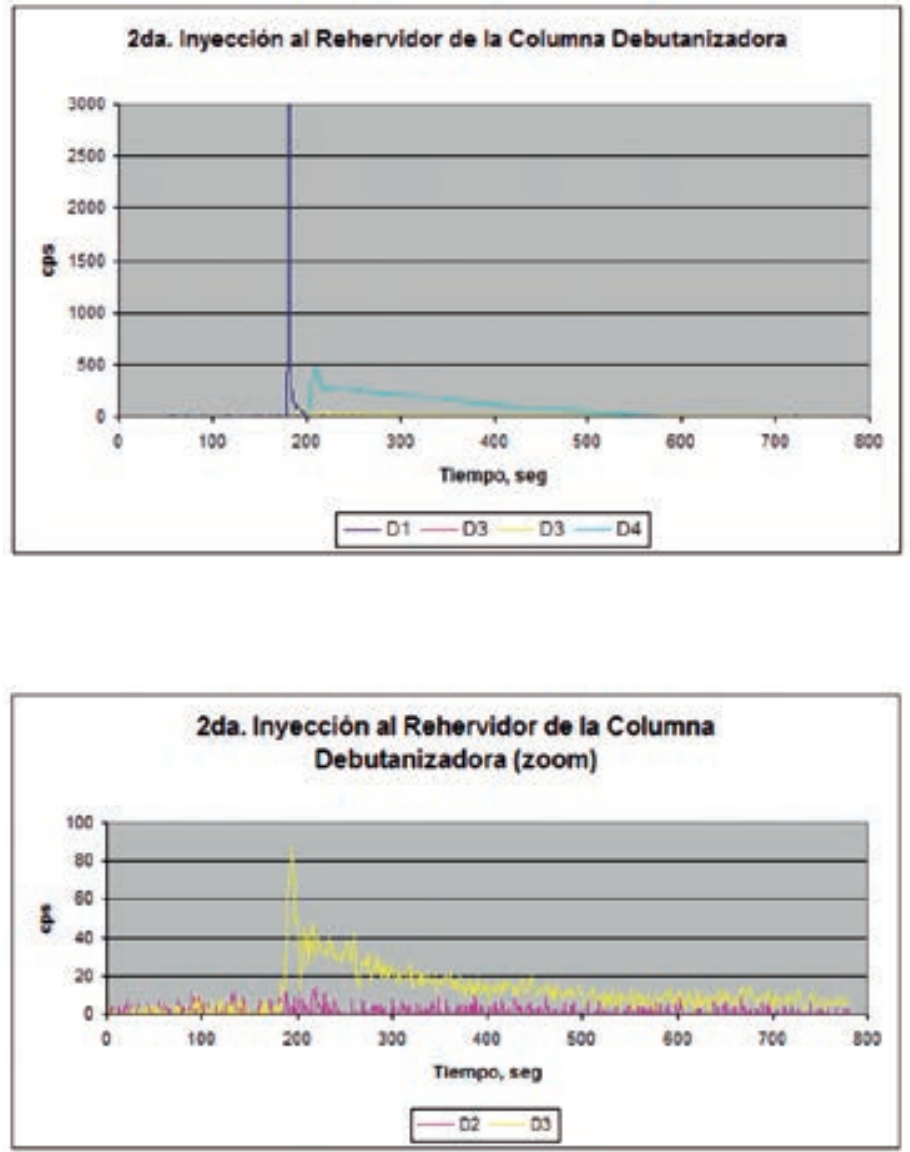


\subsection{REHERVIDOR DE LA COLUMNA DEPROPANIZADORA}

4.2.1. Primera inyección (evaluación de posibles pases de casco a tubos)
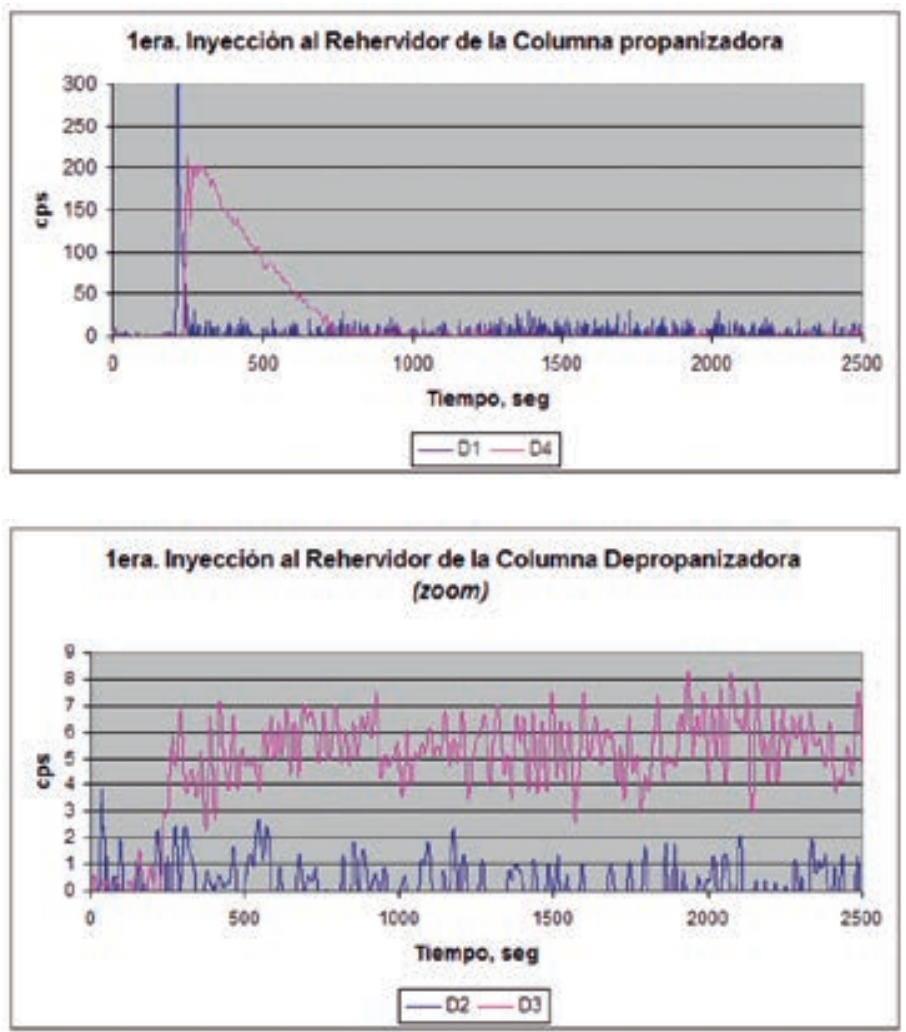

4.2.2. Segunda inyección (evaluación de posibles fugas de tubos a casco)

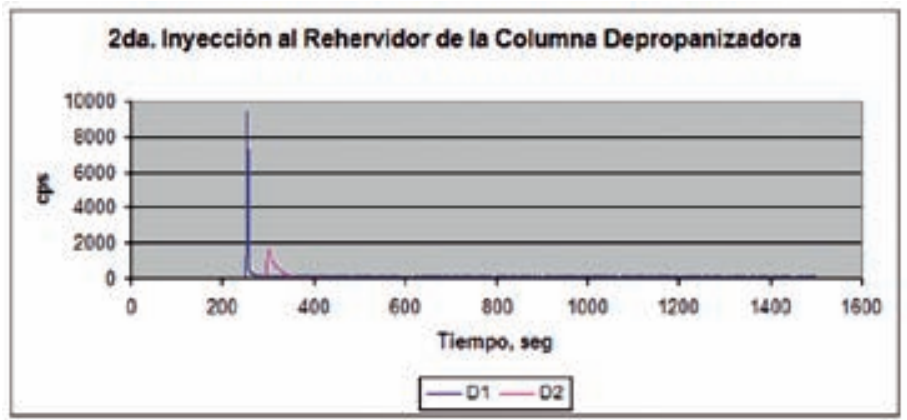




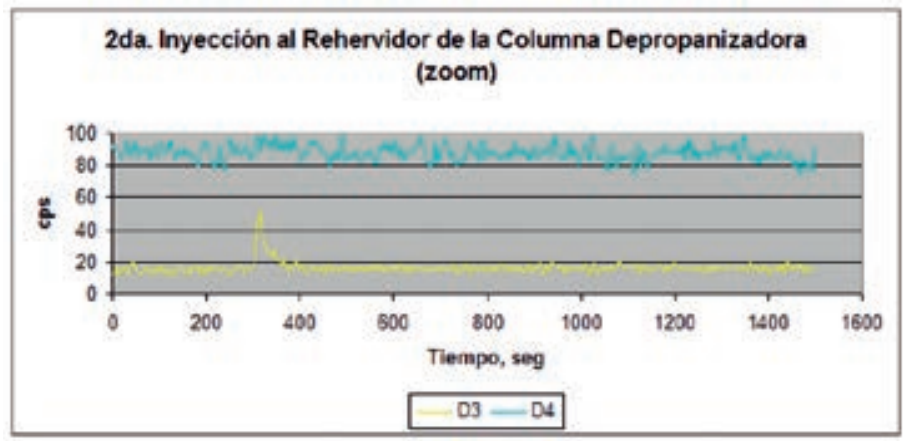

\subsection{REHERVIDOR DE COLUMNA DESPOJADORA DE LA FRACCIONADORA NAFTA-DIÉSEL}

4.3.1. Primera inyección (evaluación de posible pase de fluido térmico de casco a tubos)
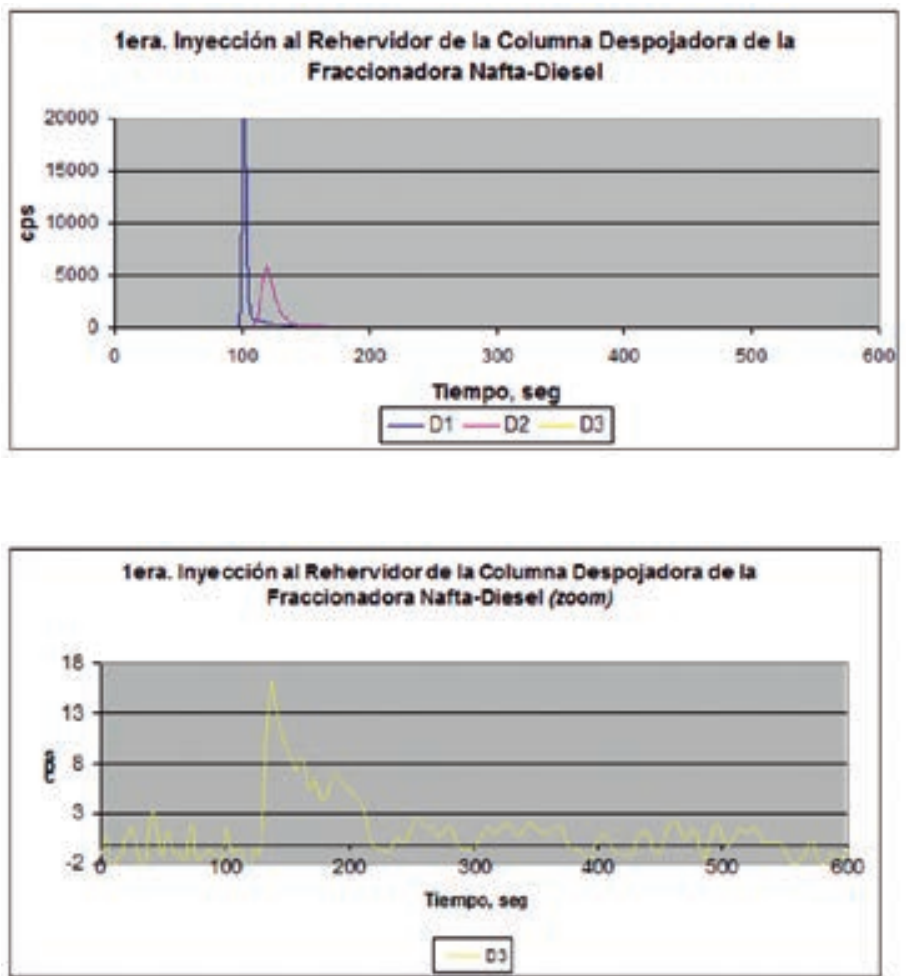


\subsubsection{Segunda inyección}
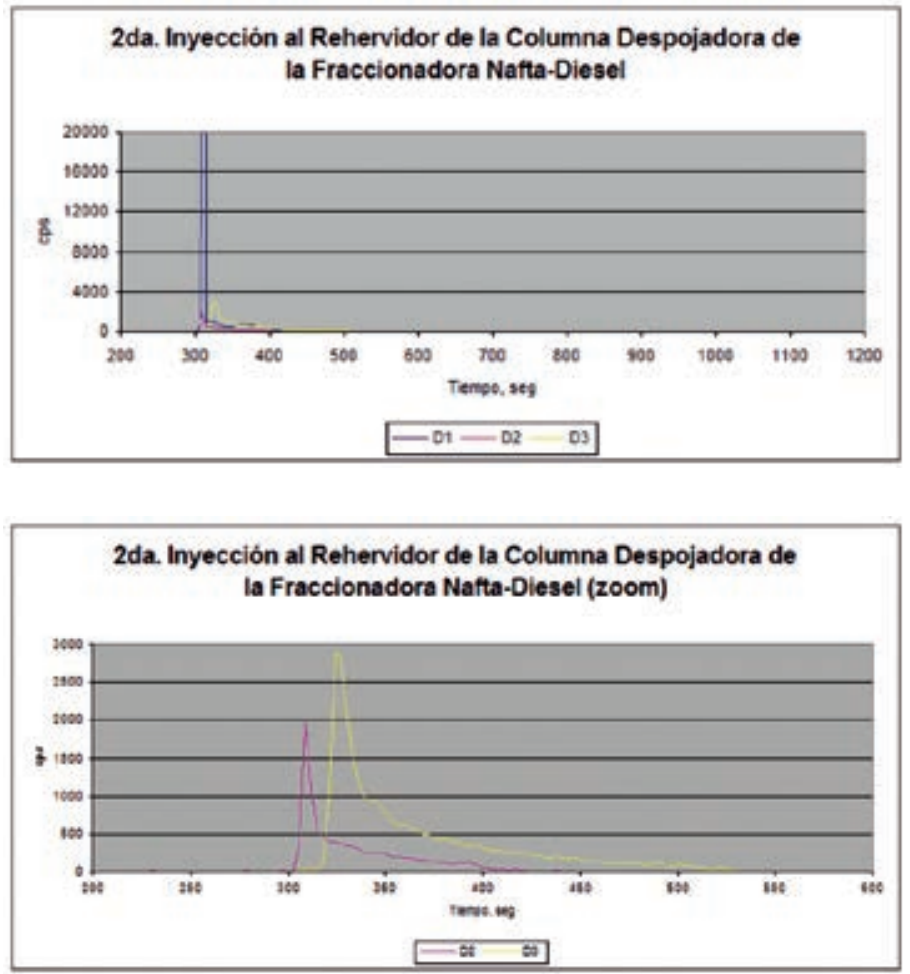

\section{ANÁLISIS Y CONCLUSIONES}

\subsection{Rehervidor de la columna debutanizadora}

Primera inyección (evaluación de posibles fugas de tubos a casco)

- La entrada del trazador a tubos corresponde a un pulso Dirac (instantáneo) sin dispersión.

- Se registra una salida de trazador por la posición del detector D4 a la salida de los fondos, por lo que hay evidencias de arrastre de fluido térmico por la corriente de fondos de la debutanizadora en el rehervidor.

Conclusión: existe fuga de tubos a casco en una magnitud de $0.4 \%$ del flujo total de fluido térmico que circula por el casco.

Segunda inyección (evaluación de posibles pases de casco a tubos)

- La entrada del trazador al casco corresponde a un pulso Dirac (instantáneo) sin dispersión.

- Luego del registro del pulso de entrada, ya no se manifiesta registro alguno en el primer detector. 
- Prácticamente el $85 \%$ de todo el trazador inyectado sale con los fondos que recorren el casco del rehervidor. El restante lo hace con el reflujo (registro del detector D3).

- No se registra ninguna salida de trazador por la posición del detector D2, a la salida del fluido térmico del rehervidor.

Conclusión: no existe pase de casco a tubos.

\subsection{REHERVIDOR DE LA COLUMNA DEPROPANIZADORA}

\section{Primera inyección (evaluación de posibles pases de casco a tubos)}

- La entrada del trazador al casco corresponde a un pulso Dirac (instantáneo) sin dispersión.

- Luego del registro del pulso de entrada, ya no se manifiesta registro alguno en el primer detector D1 hasta el final de las mediciones.

- Prácticamente todo el trazador inyectado sale con los fondos que recorren el casco del rehervidor. Una cantidad insignificante lo hace con el reflujo (registro del detector D3).

- El ligero incremento registrado en el contaje del detector D3 no está relacionado con una salida de trazador en el reflujo del rehervidor. En realidad, el D3 ha detectado cierta actividad del trazador que atraviesa por el casco del rehervidor en la vecindad más próxima.

- No se registra ninguna salida de trazador por la posición del detector D2, a la salida del fluido térmico del rehervidor.

Conclusión: no existe pase de casco a tubos.

\section{Segunda inyección (evaluación de posibles fugas de tubos a casco)}

- La entrada del trazador a tubos corresponde a un pulso Dirac (instantáneo) sin dispersión.

- No se registra ninguna salida de trazador por la posición del detector D4 a la salida de los fondos, por lo que no hay evidencias de arrastre de fluido térmico por la corriente de fondos de la depropanizadora que atraviesan el rehervidor. Solo se incrementa ligeramente el fondo natural de radiación luego de la curva de respuesta, lo cual es normal.

- El pequeño registro en el contaje del detector D3, ubicado en la línea de reflujo de fondos que salen del rehervidor, no está relacionado a una salida de trazador en dicho reflujo. En realidad, el D3 ha detectado cierta actividad del trazador que atraviesa por los tubos del rehervidor en su vecindad más próxima. Esto se confirma con ayuda de la primera experiencia, en la cual no se evidenciaron arrastres del trazador por el reflujo, incluso en condiciones en que el trazador circulaba por el casco.

Conclusión: no existen fugas de tubos a casco.

\subsection{REHERVIDOR DE COLUMNA DESPOJADORA DE LA FRACCIONADORA NAFTA-DIÉSEL}

\section{Primera inyección (evaluación de posibles pases de casco a tubos)}

- La entrada del trazador al casco, registrada por el detector D1, corresponde a un pulso poco disperso, debido al recorrido del trazador desde el punto de inyección a la válvula de purga de la línea de entrada de fluido térmico hasta la entrada a tubos. 
- Luego del registro de la función de entrada del trazador al rehervidor, ya no se manifiesta registro alguno en el primer detector D1 hasta el final de las mediciones (600 seg.). Solo quedan vestigios que incrementan ligeramente el fondo ambiental.

- Prácticamente todo el trazador inyectado sale con los fondos que recorren el casco del rehervidor. Una pequeña fracción de trazador podría estar saliendo aparentemente en el retorno de diésel a la despojadora, de acuerdo al registro del detector D3. La sospecha se desvirtúa si se consideran las diferentes escalas para cada curva de respuesta y la geometría del montaje experimental.

Como se aprecia, el registro del detector D3, aparentemente importante, es poco notorio si se establecen escalas comunes de medición para todas las curvas de respuesta.

Conclusión: no existe pase de casco a tubos.

\section{Segunda inyección (evaluación de posibles fugas de tubos a casco)}

- La entrada del trazador a tubos corresponde a un pulso Dirac (instantáneo) sin dispersión.

- Hay un registro en el contaje del detector D2 (salida del aceite térmico), por lo que estaríamos en presencia de una pequeña fuga de diésel por los tubos, la misma que sería arrastrada por el fluido térmico.

Conclusión: Se presenta fuga de diésel por los tubos del rehervidor en el tercio inferior de la longitud del aparato y con una magnitud de $0.3 \%$ del flujo total principal de diésel.

\section{REFERENCIAS BIBLIOGRÁFICAS}

[1]. D. Delacroix, J. P. Guerre y P. Leblanc, Guide Pratique Radionucléides et Radioprotection. Francia: CEA et EDP Sciences, 2004.

[2]. J. Hulla, R. Ravinger, P. Turcek, «Artificial radio-tracers in groundwater flow measurements», en Groundwater monitoring and management, Selected contributions to an International symposium at Dresden. London: University Coll, 1990, pp. 131-138.

[3]. Fundamental Safety Principles. Safety Standards Series No. SF-1. IAEA 21, 2006.

[4]. International Basic Safety Standards for Protection against Ionizing Radiation and for the Safety of Radiation Sources, IAEA Draft Safety Standard DS379, 2009.

[5]. J. P. Leclerc y G. Grevillot, «Traceurs et méthodes de traçages», Récents Progrès en Génie des procédés, 61, Vol. 12, 1998.

[6]. J. P. Leclerc, «Traceurs and tracing methods», Récents Progrès en Génie des procédés, 79, Vol. 15, 2001.

[7]. Nuclear Commision on Radiation Protection, Report No. 109 - Effects of Ionizing Radiation on Aquatic Organisms, 1991.

[8]. M. Pell y A. Wörman, «Biological Wastewater Treatment Systems», en Encyclopedia of Ecology, Ed. Brian Kronan, Países Bajos: Elsevier, 2008.

[9]. C. Sebastián, G. Maghella, E. Mamani. Y J. Maguiña, «Estudios de Dispersión de Contaminantes y Declinación Bacteriana en la Bahia del Mar de Chimbote». Instituto Peruano de Energía Nuclear. Informe Científico Tecnológico 1998-2001. Lima, Perú: IPEN, 2002, pp.257-260.

[10]. International Atomic Energy Agency, Radiotracer Applications in Industry - A Guidebook, Viena, Austria: International Atomic Energy Agency, 2004. 
INDUSTRIAL

[11]. International Atomic Energy Agency, Guidebook on Nuclear Techniques in Hydrology, Viena, Austria: IAEA, 1983.

[12]. J. Thereska, "Radiotracer Methodology and Technology», en Industrial Applications and Chemistry Section, Viena, Austria: IAEA, 1999. 